\title{
Two Patented Probiotics Mitigated the Haematological Alterations Induced by a Very Virulent Infectious Bursal Disease Virus in Chicks
}

\author{
Aliyu Danlami Andamin \\ Ahmadu Bello University \\ Paul Ayuba Abdu \\ Ahmadu Bello University \\ Felix Tersua Akade \\ College of Agriculture \\ Ochuko Orakpoghenor ( $\triangle$ ochuko.orakpoghenor@gmail.com ) \\ Ahmadu Bello University https://orcid.org/0000-0003-0833-1640 \\ Tagang Aluwong \\ Ahmadu Bello University
}

\section{Research Article}

Keywords: Mitigating, Antox ${ }^{\circledR}$, Bactofort ${ }^{\circledR}$, blood, ISA Brown, infectious bursal disease

Posted Date: March 4th, 2021

DOI: https://doi.org/10.21203/rs.3.rs-254420/v1

License: (c) (i) This work is licensed under a Creative Commons Attribution 4.0 International License. Read Full License

Version of Record: A version of this preprint was published at Veterinary Research Communications on April 22nd, 2021. See the published version at https://doi.org/10.1007/s11259-021-09790-7. 


\section{Abstract}

The study investigated the mitigating effects of two probiotics on blood parameters of ISA Brown chicks inoculated with a very virulent infectious bursal disease virus (vvIBDV). Two hundred chicks were assigned into four groups of 50 birds each. Groups $A$ and $B$ were administered $A n t o x{ }^{\circledR}$ in water and Bactofort ${ }^{\circledR}$ in feed daily from 1 to 42 days of age and inoculated with a vvIBDV at 28 days and $C$ and $D$ served as positive and negative controls, respectively. Blood samples were examined for changes in packed cell volume (PCV), haemoglobin concentration ( $\mathrm{Hb})$, red blood cell (RBC), total white blood cell (TWBC), heterophil and lymphocyte counts seven days post inoculation. The PCV between groups $A$ and $C$ differed $(P<0.05)$ and in group $B$ it was higher $(P<0.05)$ than that of group $C$. The $\mathrm{Hb}$ concentration between groups $A, B$ and $C$ differed $(P<0.05)$. There was a difference $(P<0.05)$ in $R B C$ counts between groups $A, B, C$. Differences in TWBC between group $A$ and $C$ were significant $(P<0.05)$ and TWBC in group $B$ was higher $(P<0.05)$ than that of group C. There was a significant difference in heterophil $(P<0.05)$ and lymphocyte $(P<0.05)$ count between group $A$ and $C$, and $B$ and $C$. Heterophil/lymphocyte ratio was significantly higher in positive control compared to groups $A, B, C$. Antox ${ }^{\circledR}$ and Bactofort ${ }^{\circledR}$ mitigated the deleterious effects of vvIBDV on blood parameters and can assist in the control of IBD.

\section{Introduction}

Probiotics are rich in microorganisms that improve host's health (Guillet 1998; Line et al. 1998), proteins, B-complex vitamins, trace minerals and 'Plus Factors' (Glade and Sist 1998). The functions of probiotics include enhancing availability of phosphorus and nutrient utilization, colonization or inhibition pathogens resulting in reduced incidence and duration of disease (Ehrmann et al. 2002; Moreno et al. 2002). Probiotics improve immunity by stimulating subsets of immune cells to produce cytokines, which in turn play a role in the induction and regulation of immune response and enhance epithelial innate immunity-related gene expression through anti-inflammatory cytokine (Fuller 1989; Christensen et al. 2002; Pagnini et al. 2008; Amit-Romach et al. 2010).

Infectious bursal disease (IBD) is an acute, highly contagious, immunosuppressive disease of young chickens (Abdu 2014). It is caused by a member of the genus Avian birnavirus in the family Birnaviridae, whose genome consists of double-stranded RNA segments, designated $A$ and $B$, which are enclosed within a non-enveloped icosahedral capsid (Lasher and Shane 1994; Luket and Saif, 2003; Muller et al. 2003). Two serotypes of infectious bursal disease virus (IBDV) are recognized: I and II (Jackwood et al. 1982). Only infection with serotype I viruses in chickens results in clinical disease. Serotype I viruses have been categorized into four based on pathogenicity: Classical, Variants, Attenuated and Very virulent strains (Kibenge et al. 1988). Infectious bursal disease virus (IBDV) produces two forms of disease: The sub-clinical and clinical in susceptible chickens, depending on the age of the bird at the time of infection. Sub-clinical and clinical infections with IBDV may cause immunosuppression (Lukert and Saif 2003). The sub-clinical form is observed in chicks below 3 weeks of age; while the clinical form is reported in 3-8-week old chickens (Sharma et al. 2000; Lukert and Saif 2003). Following infection, IBDV multiplies rapidly in the B-lymphocytes of the BF, leading to immunosuppression, increased susceptibility to other diseases and reduced growth rate of surviving birds (Kibenge et al. 1988; Mcllroy et al. 1989; Becht and Muller 1991). The BF is the principal organ of virus replication and peak virus titres in the BF can be detected between 3 and 5 days after IBDV infection (Lukert and Saif 1997). Uncomplicated IBDV infection with original type I isolates results in up to $90 \%$ flock morbidity and $20 \%$ mortality in susceptible $3-8$ weeks-old hybrid Leghorn replacement pullets (Lasher and Shane 1994).

Haematology evaluates the health, physiological and biological status of birds (Ross et al., 1976). It checks for haemoglobin and haematocrit (evaluation of anaemia), rate of leucocytes or white blood cells (infection indicators) and heterophil/lymphocyte ratio (stress indicator) (Oladele et al. 2005). Blood components may be influenced by physiological factors, such as age and species, and pathological factors (Cheneme and Cho, 1984). There is no 
information on the effects of probiotics on haematological parameters of commercial chickens inoculated with a vVIBDV.

\section{Materials And Methods}

\section{Experimental chicks and housing}

Two hundred day-old ISA Brown pullet chicks were purchased from a commercial hatchery, housed on deep litter and provided a floor space of 0.10 square metres per bird. Before stocking the house was cleaned, washed and disinfected. Rodent and insect control was achieved using a rodenticide and insecticide, respectively twice one week apart.

\section{Feeds and feeding}

Chick mash was purchased from a commercial feed distributor in Zaria, Nigeria and proximate analyses carried out. The feed contained the following nutrients: \% DM 97.20, \% ASH 13.96, \% EE 7.41, \% CF 6.49, \% N 3.60 and \% CP 22 .50. All the chicks were fed with for up to 42 days of age. The chicks were allowed access to feed and water ad libitum.

\section{Probiotics}

Liquid Antox ${ }^{\circledR}$ containing, Saccharomyces cerevisiae $\left(4.125 \times 10^{6} \mathrm{cfu} / \mathrm{mL}\right)$, Citric acid- $6 \mathrm{~g}$, Lactic acid-2 $\mathrm{g}$, Vitamins $\mathrm{B}_{1^{-}}$ $100 \mathrm{mg}, \mathrm{B}_{2}-7.5 \mathrm{mg}, \mathrm{B}_{6}-80 \mathrm{mg}$, and $\mathrm{B}_{12}-0.6 \mathrm{mg}$, Biotin-1.5 mg, Nicotinamide- $1 \mathrm{~g}$, Calcium chrorine-300 mg, Potassium iodide-4.6 mg, Sodium selenite-78.8 mg, Zinc chloride-320 mg, Iron chloride-300 mg, Magnesium chloride hexahydrate250 mg, Manganese chloride-631 mg, Copper sulphate-32 mg, Cobalt chloride-3.08 mg, manufactured by (Montajat

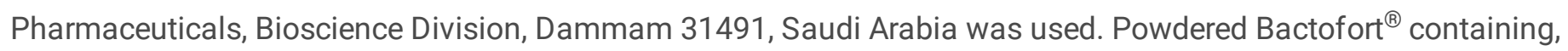
Lactobacillus acidophilus $\left(77 \times 10^{9} \mathrm{cfu} / \mathrm{kg}\right)$, Enterococcus faecium $\left(44 \times 10^{9} \mathrm{cfu} / \mathrm{kg}\right)$, Saccharomyces cerevisiae $(5,000$ $\left.\times 10^{9} \mathrm{cells} / \mathrm{kg}\right)$, Bacillus subtilis $\left(2.2 \times 10^{9} \mathrm{cfu} / \mathrm{kg}\right)$, manufactured by (Biofeed Technology Inc., Brossand, QC, Canada was used.

\section{Inoculation of chicks with infectious bursal disease virus}

A characterised vvIBDV [24] was obtained from the Department of Veterinary Medicine, Faculty of Veterinary Medicine, Ahmadu Bello University, Zaria, Nigeria. Each chick in the test and positive groups was inoculated with $0.05 \mathrm{ml}$ of a vvIBDV suspension $\left(10^{-9.76} \mathrm{CID}_{50} / \mathrm{mL}\right)$ via conjunctival instillation at 28 days of age.

\section{Experimental design}

Two hundred day old ISA Brown chicks were assigned randomly into four groups, A, B, C and D with 50 chicks each. Group A was administered Anthox ${ }^{\circledR}$ at $1.5 \mathrm{~mL} / \mathrm{L}$ in drinking water daily from day-old to 42 days of age and inoculated with a vvIBDV at 28 days of age ( 0 dpi). Group B chicks were administered Bactofort ${ }^{\circledR}$ at $12.5 \mathrm{~g} / 25 \mathrm{~kg}$ in feed daily from day-old to 42 days of age and inoculated with a vvIBDV at 28 days of age. No probiotic was administered to chicks in group $\mathrm{C}$ but were inoculated with a vvIBDV at 28 days of age and. chicks in group $\mathrm{D}$ were not administered any probiotic and not inoculated with vvIBDV.

\section{Collection of blood}

Blood was collected through the heart from each bird at weekly interval from day-old to 42 days of age. The blood was emptied into a heparinised universal bottle. Blood samples were labeled and processed for haematology (Campbell and Ellis 2007). 


\section{Data analyses}

\section{Data collected were presented as means \pm standard errors of the mean (Mean \pm SEM). Two-way analysis of variance (ANOVA) was used in the analyses of the data. Bonferoni's multiple comparison post-hoc tests was used to analysed the level of significance at $\mathrm{P}<$ 0.05. GraphPad Prism 4.0 for windows (GraphPad Software, San Diego, California USA) was used to conduct the analyses.}

\section{Results}

\section{Packed cell volume}

The PCV in groups A and B decreased from a value of $30.40 \pm 0.11 \%$, and $28.20 \pm 0.58 \%$ at 0 -day post inoculation ( 0 dpi) with vvIBDV to $19.30 \pm 0.37 \%$, and $17.00 \pm 0.35 \%$ at $7 \mathrm{dpi}$, respectively but the decrease was lower compared to values for group C $(25.80 \pm 0.69 \%$, and $10.20 \pm 0.37 \%)$. There was a significant difference $(P<0.05)$ in the PCV between $\mathrm{A}, \mathrm{B}$ and $\mathrm{C}$ at $7 \mathrm{dpi}$ (Table 1).

\section{Haemoglobin concentration}

The mean $\mathrm{Hb}$ concentration of groups $\mathrm{A}$ and $\mathrm{B}$ decreased from mean value of $9.76 \pm 0.71 \mathrm{~g} / \mathrm{dl}$, and $9.02 \pm 0.19 \mathrm{~g} / \mathrm{dl}$ to $6.16 \pm 0.14 \mathrm{~g} / \mathrm{dl}$, and $6.00 \pm 0.27 \mathrm{~g} / \mathrm{dl}$, respectively, compared to group C $(4.20 \pm 0.25 \mathrm{~g} / \mathrm{dl})$ at $7 \mathrm{dpi}$. There was a significant difference $(P<0.05)$ in $\mathrm{Hb}$ value between groups $A, B$ and $C$ at 7 dpi (Table 2).

\section{Red blood cells and leucocyte counts}

The RBC count of groups A and B decreased from $7.55 \pm 0.62 \times 10^{12} / \mathrm{L}$ and $6.95 \pm 0.16 \times 10^{12} / \mathrm{L}$ at 0 dpi to $5.40 \pm 0.29$ $\times 10^{12} / \mathrm{L}$ and $4.10 \pm 0.43 \times 10^{12} / \mathrm{L}$ at $7 \mathrm{dpi}$ but the decrease was significantly $(P<0.05)$ lower compared to group $C(3.40$ $\left.\pm 0.43 \times 10^{12} / \mathrm{L}\right)$ at $7 \mathrm{dpi}$. There was a significant difference $(P<0.05)$ in the RBC count between $A, B$ and $C$ at $7 \mathrm{dpi}$ (Table 3). The TWBC count of groups A and B decreased from $13.88 \pm 0.24 \times 10^{9} / \mathrm{L}$ and $12.58 \pm 0.32 \times 10^{9} / \mathrm{L}$ at 0 dpi to $8.90 \pm 0.29 \times 10^{9} / \mathrm{L}$ and $7.00 \pm 0.42 \times 10^{9} / \mathrm{L}$ at $7 \mathrm{dpi}$ compared to group $\mathrm{C}\left(4.30 \pm 0.37 \times 10^{9} / \mathrm{L}\right)$ at $7 \mathrm{dpi}$. There was a significant difference $(P<0.001)$ in the TWBC counts between $A, B$ and $C$ at 7 dpi (Table 4).

\section{Differential leucocyte counts}

The heterophil counts of the groups (A and B) administered probiotics and inoculated with vvIBDV decreased from 4.09 $\pm 0.02 \times 10^{9} / \mathrm{l}$, and $4.05 \pm 0.02 \times 10^{9} / \mathrm{l}$, at $0 \mathrm{dpi}$ to $3.04 \pm 0.02 \times 10^{9} / \mathrm{I}$ and $3.00 \pm 0.03 \times 10^{9} / \mathrm{I}$ at $7 \mathrm{dpi}$, respectively but the decrease was significantly lower compared to positive control (group C) $\left(2.98 \pm 0.02 \times 10^{9} / \mathrm{I}\right)$ at $7 \mathrm{dpi}$. There was a significant difference $(P<0.001)$ in the heterophils count between groups $A, B$ and $C$ at 7 dpi (Table 5). Lymphocyte counts of groups $A$ and $B$ decreased from $7.05 \pm 0.01 \times 10^{9} / \mathrm{I}$, and $7.02 \pm 0.02 \times 10^{9} / \mathrm{I}$ at 0 dpi to $5.05 \pm 0.00 \times 10^{9} / \mathrm{I}$ and $4.55 \pm 0.00 \times 10^{9} / \mathrm{I}$ at $7 \mathrm{dpi}$, respectively but the decreases were significantly lower compared to positive control group $\mathrm{C}$ $\left(3.04 \pm 0.02 \times 10^{9} / \mathrm{I}\right)$ at $7 \mathrm{dpi}$. There was a significant difference $(\mathrm{P}<0.05)$ in the lymphocyte count between groups $\mathrm{A}, \mathrm{B}$ and $\mathrm{C}$ at 7 dpi (Table 6). 
There were increases in heterophil/lymphocyte ratios in groups $A, B$, and $C(0.75 \pm 0.00,0.84 \pm 0.00$ and $0.98 \pm 0.10)$ at 7 dpi with vvIBDV. However, the increase was significantly lower in the groups $A$ and $B$ compared to positive control group $(C)$. There was statistical significant difference $(P<0.05)$ in the heterophil/lymphocyte ratios between group $A, B$ and $C$ at $7 \mathrm{dpi}$ (Table 7).

Table 1: Mean ( \pm SE) packed cell volume $(\%)$ of ISA Brown chicks $(n=5)$ administered Antox ${ }^{\circledR}$ and Bactofort ${ }^{\circledR}$ from day old and inoculated with a very virulent infectious bursal disease virus at 28 days of age

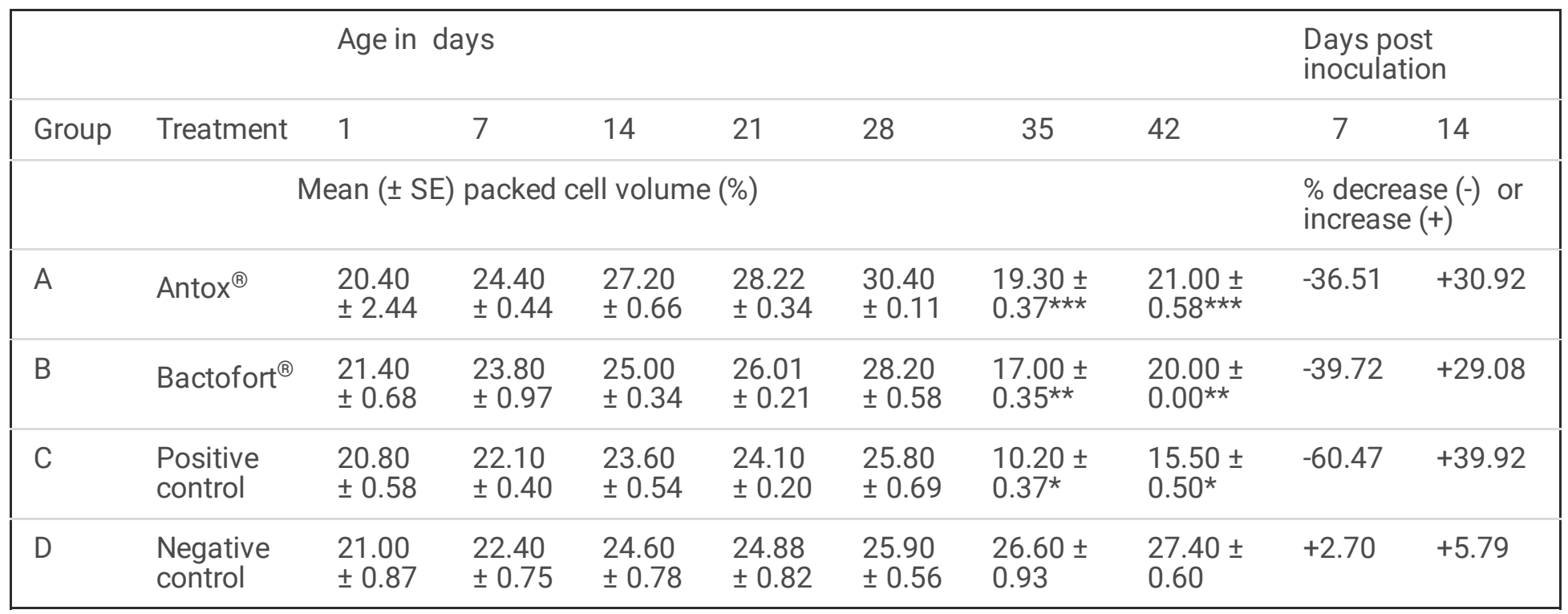

Key: $n=$ number of birds sampled, $S E=$ standard error of mean, means with asterics $(*)(P<0.05),(* \star)(P<0.01)$, ( $\left.{ }^{\star \star \star}\right)$, $(P<0.001)$ in the same column differed significantly

Table 2: Mean ( \pm SE) haemoglobin concentration $(\mathrm{g} / \mathrm{dl})$ of ISA Brown chicks $(\mathrm{n}=5)$ administered Antox ${ }^{\circledR}$ and Bactofort ${ }^{\circledR}$ from day old and inoculated with a very virulent infectious bursal disease virus at 28 days of age

\begin{tabular}{|c|c|c|c|c|c|c|c|c|c|c|}
\hline \multirow[b]{2}{*}{ Group } & \multirow[b]{2}{*}{ Treatment } & \multicolumn{7}{|c|}{ Age in days } & \multicolumn{2}{|c|}{$\begin{array}{l}\text { Days post } \\
\text { inoculation }\end{array}$} \\
\hline & & 1 & 7 & 14 & 21 & 28 & 35 & 42 & 7 & 14 \\
\hline & \multicolumn{8}{|c|}{ Mean ( \pm SE) haemoglobin concentration $(\mathrm{g} / \mathrm{dl})$} & \multicolumn{2}{|c|}{$\begin{array}{l}\% \text { decrease }(-) \text { or } \\
\text { increase }(+)\end{array}$} \\
\hline A & Antox ${ }^{\circledR}$ & $\begin{array}{l}7.04 \pm \\
0.81\end{array}$ & $\begin{array}{l}7.74 \pm \\
0.81\end{array}$ & $\begin{array}{l}8.80 \pm \\
0.30\end{array}$ & $\begin{array}{l}9.38 \pm \\
0.42\end{array}$ & $\begin{array}{l}9.76 \pm \\
0.71\end{array}$ & $\begin{array}{l}6.16 \pm \\
0.14^{\star \star \star}\end{array}$ & $\begin{array}{l}7.37 \pm \\
0.52^{\star \star \star}\end{array}$ & -36.89 & +24.49 \\
\hline $\mathrm{B}$ & Bactofort $^{\circledR}$ & $\begin{array}{l}7.05 \pm \\
0.23\end{array}$ & $\begin{array}{l}7.76 \pm \\
0.23\end{array}$ & $\begin{array}{l}8.62 \pm \\
0.44\end{array}$ & $\begin{array}{l}8.90 \pm \\
0.55\end{array}$ & $\begin{array}{l}9.02 \pm \\
0.19\end{array}$ & $\begin{array}{l}6.00 \pm \\
0.27 \star \star\end{array}$ & $\begin{array}{l}7.00 \pm \\
0.00 \star \star\end{array}$ & -33.48 & +22.39 \\
\hline $\mathrm{C}$ & $\begin{array}{l}\text { Positive } \\
\text { control }\end{array}$ & $\begin{array}{l}7.04 \pm \\
0.17\end{array}$ & $\begin{array}{l}7.10 \pm \\
0.29\end{array}$ & $\begin{array}{l}7.16 \pm \\
0.52\end{array}$ & $\begin{array}{l}7.25 \pm \\
0.62\end{array}$ & $\begin{array}{l}7.36 \pm \\
0.56\end{array}$ & $\begin{array}{l}4.20 \pm \\
0.25^{\star}\end{array}$ & $\begin{array}{l}5.75 \pm \\
0.25^{\star}\end{array}$ & -42.93 & +21.88 \\
\hline $\mathrm{D}$ & $\begin{array}{l}\text { Negative } \\
\text { control }\end{array}$ & $\begin{array}{l}7.03 \pm \\
0.61\end{array}$ & $\begin{array}{l}7.08 \pm \\
0.55\end{array}$ & $\begin{array}{l}7.16 \pm \\
0.29\end{array}$ & $\begin{array}{l}7.38 \pm \\
0.30\end{array}$ & $\begin{array}{l}7.46 \pm \\
0.21\end{array}$ & $\begin{array}{l}7.66 \pm \\
0.31\end{array}$ & $\begin{array}{l}7.88 \pm \\
0.53\end{array}$ & +2.62 & +5.63 \\
\hline
\end{tabular}

Key: $n=$ number of birds sampled, $S E=$ standard error of mean, Means with asterics $(*)(P<0.05),(* \star)(P<0.01),(* \star \star)$, $(P<0.001)$ in the same column differed significantly 
Table 3: Mean $\left( \pm\right.$ SE) red blood cell count $\left(x 10^{12} / \mathrm{I}\right)$ of ISA Brown chicks $(n=5)$ administered Antox ${ }^{\circledR}$ and Bactofort ${ }^{\circledR}$ from day old and inoculated with a very virulent infectious bursal disease virus at 28 days of age

\begin{tabular}{|c|c|c|c|c|c|c|c|c|c|c|}
\hline \multirow[b]{2}{*}{ Group } & \multirow[b]{2}{*}{ Treatment } & \multicolumn{7}{|c|}{ Age in days } & \multicolumn{2}{|c|}{$\begin{array}{l}\text { Days post } \\
\text { inoculation }\end{array}$} \\
\hline & & 1 & 7 & 14 & 21 & 28 & 35 & 42 & 7 & 14 \\
\hline & \multicolumn{8}{|c|}{ Mean $\left( \pm\right.$ SE) red blood cell count $\left(\times 10^{12} / \mathrm{I}\right)$} & \multicolumn{2}{|c|}{$\begin{array}{l}\% \text { decrease }(-) \text { or } \\
\text { increase }(+)\end{array}$} \\
\hline$A$ & Antox ${ }^{\circledR}$ & $\begin{array}{l}4.33 \pm \\
0.48\end{array}$ & $\begin{array}{l}6.80 \pm \\
0.62\end{array}$ & $\begin{array}{l}6.99 \pm \\
0.27\end{array}$ & $\begin{array}{l}7.10 \pm \\
0.26\end{array}$ & $\begin{array}{l}7.55 \pm \\
0.62\end{array}$ & $\begin{array}{l}5.40 \pm \\
0.29 * \star \star\end{array}$ & $\begin{array}{l}7.00 \pm \\
0.29 * \star \star\end{array}$ & -28.48 & +7.28 \\
\hline B & Bactofort $^{\circledR}$ & $\begin{array}{l}4.32 \pm \\
0.86\end{array}$ & $\begin{array}{l}6.50 \pm \\
0.35\end{array}$ & $\begin{array}{l}6.75 \pm \\
0.19\end{array}$ & $\begin{array}{l}6.85 \pm \\
0.21\end{array}$ & $\begin{array}{l}6.95 \pm \\
0.16\end{array}$ & $\begin{array}{l}4.10 \pm \\
0.43^{\star \star}\end{array}$ & $\begin{array}{l}6.00 \pm \\
0.00 * \star\end{array}$ & -41.01 & +13.67 \\
\hline $\mathrm{C}$ & $\begin{array}{l}\text { Positive } \\
\text { control }\end{array}$ & $\begin{array}{l}4.31 \pm \\
0.54\end{array}$ & $\begin{array}{l}5.40 \pm \\
0.29\end{array}$ & $\begin{array}{l}5.50 \pm \\
0.51\end{array}$ & $\begin{array}{l}5.65 \pm \\
0.75\end{array}$ & $\begin{array}{l}5.78 \pm \\
0.63\end{array}$ & $\begin{array}{l}3.40 \pm \\
0.43^{*}\end{array}$ & $\begin{array}{l}4.25 \pm \\
0.25^{\star}\end{array}$ & -41.18 & +26.47 \\
\hline D & $\begin{array}{l}\text { Negative } \\
\text { control }\end{array}$ & $\begin{array}{l}4.32 \pm \\
0.29\end{array}$ & $\begin{array}{l}5.30 \pm \\
0.25\end{array}$ & $\begin{array}{l}5.40 \pm \\
0.94\end{array}$ & $\begin{array}{l}5.58 \pm \\
0.99\end{array}$ & $\begin{array}{l}5.70 \pm \\
0.60\end{array}$ & $\begin{array}{l}6.30 \pm \\
0.12\end{array}$ & $\begin{array}{l}6.52 \pm \\
0.37\end{array}$ & +10.53 & +14.39 \\
\hline
\end{tabular}

Key: $n=$ number of birds sampled, $S E=$ standard error of the mean, means with asterics $(*)(P<0.05),\left({ }^{\star \star}\right)(P<0.01)$, $(\star \star \star),(P<0.001)$ in the same column differed significantly

Table 4: Mean ( \pm SE) total white blood cell count $\left(x 10^{9} / \mathrm{I}\right)$ of ISA Brown chicks $(n=5)$ administered Antox ${ }^{\circledR}$ and Bactofort ${ }^{\circledR}$ from day old and inoculated with a very virulent infectious bursal disease virus at 28 days of age

\begin{tabular}{|c|c|c|c|c|c|c|c|c|c|c|}
\hline \multirow[b]{2}{*}{ Group } & \multirow[b]{2}{*}{ Treatment } & \multicolumn{7}{|c|}{ Age in days } & \multicolumn{2}{|c|}{$\begin{array}{l}\text { Days post } \\
\text { inoculation }\end{array}$} \\
\hline & & 1 & 7 & 14 & 21 & 28 & 35 & 42 & 7 & 14 \\
\hline & & \multicolumn{7}{|c|}{ Mean $\left( \pm\right.$ SE) total white blood cell count $\left(\times 10^{9} / \mathrm{I}\right)$} & \multicolumn{2}{|c|}{$\begin{array}{c}\text { \% decrease (-) } \\
\text { or increase }(+)\end{array}$} \\
\hline A & Antox ${ }^{\circledR}$ & $\begin{array}{l}8.06 \\
\pm \\
0.61\end{array}$ & $\begin{array}{l}10.95 \\
\pm 0.94\end{array}$ & $\begin{array}{l}11.96 \\
\pm 0.74\end{array}$ & $\begin{array}{l}12.96 \\
\pm 0.85\end{array}$ & $\begin{array}{l}13.88 \\
\pm 0.24\end{array}$ & $\begin{array}{l}8.90 \pm \\
0.29 * \star \star\end{array}$ & $\begin{array}{l}10.00 \pm \\
0.29 * \star \star\end{array}$ & -35.88 & +27.95 \\
\hline B & Bactofort $^{\circledR}$ & $\begin{array}{l}8.07 \\
\pm \\
0.59\end{array}$ & $\begin{array}{l}9.75 \pm \\
0.74\end{array}$ & $\begin{array}{l}10.85 \\
\pm 0.35\end{array}$ & $\begin{array}{l}11.62 \\
\pm 0.22\end{array}$ & $\begin{array}{l}12.58 \\
\pm 0.32\end{array}$ & $\begin{array}{l}7.00 \pm \\
0.42^{\star \star}\end{array}$ & $\begin{array}{l}9.00 \pm \\
0.00 * \star\end{array}$ & -44.35 & +28.46 \\
\hline C & $\begin{array}{l}\text { Positive } \\
\text { control }\end{array}$ & $\begin{array}{l}8.06 \\
\pm \\
0.94\end{array}$ & $\begin{array}{l}8.23 \pm \\
0.69\end{array}$ & $\begin{array}{l}8.44 \pm \\
0.73\end{array}$ & $\begin{array}{l}8.98 \pm \\
0.25\end{array}$ & $\begin{array}{l}9.20 \pm \\
0.54\end{array}$ & $\begin{array}{l}4.30 \pm \\
0.37{ }^{\star}\end{array}$ & $\begin{array}{l}6.00 \pm \\
0.50 \star\end{array}$ & -53.26 & +34.78 \\
\hline D & $\begin{array}{l}\text { Negative } \\
\text { control }\end{array}$ & $\begin{array}{l}8.06 \\
\pm \\
0.38\end{array}$ & $\begin{array}{l}8.22 \pm \\
0.58\end{array}$ & $\begin{array}{l}8.42 \pm \\
0.54\end{array}$ & $\begin{array}{l}8.99 \pm \\
0.64\end{array}$ & $\begin{array}{l}9.18 \pm \\
0.44\end{array}$ & $\begin{array}{l}9.48 \pm \\
0.25\end{array}$ & $\begin{array}{l}9.80 \pm \\
0.07\end{array}$ & +3.27 & +6.75 \\
\hline
\end{tabular}

Key: $n=$ number of birds sampled, $S E=$ standard error of the mean, means with asterics $(*)(P<0.05),(* *)(P<0.01)$, $(\star * *),(P<0.001)$ in the same column differed significantly

Table 5: Mean ( \pm SE) heterophil count $\left(x 10^{9} / \mathrm{I}\right)$ of ISA Brown chicks $(n=5)$ administered Antox ${ }^{\circledR}$ and Bactofort ${ }^{\circledR}$ from day old and inoculated with a very virulent infectious bursal disease virus at 28 days of age 


\begin{tabular}{|c|c|c|c|c|c|c|c|c|c|c|}
\hline \multicolumn{9}{|c|}{ Age in days } & \multicolumn{2}{|c|}{$\begin{array}{l}\text { Days post } \\
\text { inoculation }\end{array}$} \\
\hline Group & Treatment & 1 & 7 & 14 & 21 & 28 & 35 & 42 & 7 & 14 \\
\hline \multicolumn{9}{|c|}{ Mean ( \pm SE) heterophil count $\left(\times 10^{9} / \mathrm{I}\right)$} & \multicolumn{2}{|c|}{$\begin{array}{l}\text { \% decrease }(-) \text { or } \\
\text { increase }(+)\end{array}$} \\
\hline$A$ & Antox $^{\circledR}$ & $\begin{array}{l}2.05 \pm \\
0.01\end{array}$ & $\begin{array}{l}2.95 \pm \\
0.02\end{array}$ & $\begin{array}{l}3.33 \pm \\
0.00\end{array}$ & $\begin{array}{l}3.75 \pm \\
0.02\end{array}$ & $\begin{array}{l}4.09 \pm \\
0.02\end{array}$ & $\begin{array}{l}3.04 \pm \\
0.02 \star \star \star\end{array}$ & $\begin{array}{l}3.45 \pm \\
0.01^{\star \star \star}\end{array}$ & -25.67 & +15.65 \\
\hline B & Bactofort $^{\circledR}$ & $\begin{array}{l}2.06 \pm \\
0.02\end{array}$ & $\begin{array}{l}3.88 \pm \\
0.01\end{array}$ & $\begin{array}{l}3.25 \pm \\
0.02\end{array}$ & $\begin{array}{l}3.70 \pm \\
0.00\end{array}$ & $\begin{array}{l}4.05 \pm \\
0.02\end{array}$ & $\begin{array}{l}3.00 \pm \\
0.03^{\star \star}\end{array}$ & $\begin{array}{l}3.20 \pm \\
0.01 * \star\end{array}$ & -25.93 & +20.99 \\
\hline C & $\begin{array}{l}\text { Positive } \\
\text { control }\end{array}$ & $\begin{array}{l}2.05 \pm \\
0.02\end{array}$ & $\begin{array}{l}2.51 \pm \\
0.03\end{array}$ & $\begin{array}{l}2.88 \pm \\
0.01\end{array}$ & $\begin{array}{l}3.26 \pm \\
0.03\end{array}$ & $\begin{array}{l}3.56 \pm \\
0.02\end{array}$ & $\begin{array}{l}2.98 \pm \\
0.02^{\star}\end{array}$ & $\begin{array}{l}3.05 \pm \\
0.03^{*}\end{array}$ & -16.29 & +14.33 \\
\hline D & $\begin{array}{l}\text { Negative } \\
\text { control }\end{array}$ & $\begin{array}{l}2.06 \pm \\
0.01\end{array}$ & $\begin{array}{l}2.52 \pm \\
0.01\end{array}$ & $\begin{array}{l}2.81 \pm \\
0.02\end{array}$ & $\begin{array}{l}3.25 \pm \\
0.03\end{array}$ & $\begin{array}{l}3.55 \pm \\
0.01\end{array}$ & $\begin{array}{l}3.82 \pm \\
0.00\end{array}$ & $\begin{array}{l}4.14 \pm \\
0.02\end{array}$ & +7.61 & +16.62 \\
\hline
\end{tabular}

Key: $n=$ number of birds sampled, $S E=$ standard error of the mean, means with asterics $\left({ }^{\star}\right)(P<0.05),\left({ }^{\star \star}\right)(P<0.01)$, $(\star * *),(P<0.001)$ in the same column differed significantly

Table 6: Mean ( \pm SE) lymphocyte count $\left(x 10^{9} /\right.$ I) of ISA Brown chicks $(n=5)$ administered Antox ${ }^{\circledR}$ and Bactofort ${ }^{\circledR}$ from day old and inoculated with a very virulent infectious bursal disease virus at 28 days of age

\begin{tabular}{|c|c|c|c|c|c|c|c|c|c|c|}
\hline \multicolumn{8}{|c|}{ Age in days } & \multicolumn{3}{|c|}{ Days post inoculation } \\
\hline Group & Treatment & & 7 & 14 & 21 & 28 & 35 & 42 & 7 & 14 \\
\hline \multicolumn{9}{|c|}{ Mean ( \pm SE) lymphocyte count $\left(\times 10^{9} / \mathrm{I}\right)$} & \multicolumn{2}{|c|}{$\%$ decrease $(-)$ or increase $(+)$} \\
\hline$A$ & Antox ${ }^{\circledR}$ & $\begin{array}{l}3.77 \\
\pm \\
0.00\end{array}$ & $\begin{array}{l}4.99 \\
\pm \\
0.01\end{array}$ & $\begin{array}{l}5.77 \\
\pm \\
0.00\end{array}$ & $\begin{array}{l}6.65 \\
\pm \\
0.01\end{array}$ & $\begin{array}{l}7.05 \\
\pm \\
0.01\end{array}$ & $\begin{array}{l}5.05 \pm \\
0.00 \star \star \star\end{array}$ & $\begin{array}{l}4.85 \pm \\
0.03^{\star \star \star}\end{array}$ & -42.55 & +31.21 \\
\hline B & Bactofort $^{\circledR}$ & $\begin{array}{l}3.78 \\
\pm \\
0.03\end{array}$ & $\begin{array}{l}4.85 \\
\pm \\
0.04\end{array}$ & $\begin{array}{l}5.65 \\
\pm \\
0.01\end{array}$ & $\begin{array}{l}6.59 \\
\pm \\
0.02\end{array}$ & $\begin{array}{l}7.02 \\
\pm \\
0.02\end{array}$ & $\begin{array}{l}4.55 \pm \\
0.00 \star \star\end{array}$ & $\begin{array}{l}3.99 \pm \\
0.00 * \star\end{array}$ & -49.43 & +43.16 \\
\hline $\mathrm{C}$ & $\begin{array}{l}\text { Positive } \\
\text { control }\end{array}$ & $\begin{array}{l}3.77 \\
\pm \\
0.01\end{array}$ & $\begin{array}{l}3.99 \\
\pm \\
0.01\end{array}$ & $\begin{array}{l}4.57 \\
\pm \\
0.03\end{array}$ & $\begin{array}{l}4.86 \\
\pm \\
0.03\end{array}$ & $\begin{array}{l}5.73 \\
\pm \\
0.01\end{array}$ & $\begin{array}{l}3.04 \pm \\
0.02 \star\end{array}$ & $\begin{array}{l}3.25 \pm \\
0.00 *\end{array}$ & -46.95 & +43.28 \\
\hline D & $\begin{array}{l}\text { Negative } \\
\text { control }\end{array}$ & $\begin{array}{l}3.78 \\
\pm \\
0.00\end{array}$ & $\begin{array}{l}3.98 \\
\pm \\
0.04\end{array}$ & $\begin{array}{l}4.61 \\
\pm \\
0.01\end{array}$ & $\begin{array}{l}4.95 \\
\pm \\
0.01\end{array}$ & $\begin{array}{l}5.82 \\
\pm \\
0.01\end{array}$ & $\begin{array}{l}6.54 \pm \\
0.02\end{array}$ & $\begin{array}{l}7.32 \pm \\
0.01\end{array}$ & +12.37 & +25.77 \\
\hline
\end{tabular}

Key: $n=$ number of birds sampled, $S E=$ standard error of the mean, means with asterics $(*)(P<0.05),\left({ }^{\star *}\right)(P<0.01)$, $(\star \star *)(P<0.001)$ in the same column differed significantly

Table 7: Mean ( \pm SE) heterophil/lymphocyte ratio of ISA Brown chicks $(n=5)$ administered Antox ${ }^{\circledR}$ and Bactofort ${ }^{\circledR}$ from day old and inoculated with a very virulent infectious bursal disease virus at 28 days of age 


\begin{tabular}{|c|c|c|c|c|c|c|c|c|c|c|}
\hline \multirow[b]{2}{*}{ Group } & \multirow[b]{2}{*}{ Treatment } & \multicolumn{7}{|c|}{ Age in days } & \multicolumn{2}{|c|}{ Days post inoculation } \\
\hline & & 1 & 7 & 14 & 21 & 28 & 35 & 42 & 7 & 14 \\
\hline & \multicolumn{8}{|c|}{ Mean $( \pm$ SE) heterophil/lymphocyte ratio } & \multicolumn{2}{|c|}{$\begin{array}{l}\text { \% increase }(-) \text { or decrease } \\
(+)\end{array}$} \\
\hline$A$ & Antox ${ }^{\circledR}$ & $\begin{array}{l}0.53 \\
\pm \\
0.00\end{array}$ & $\begin{array}{l}0.59 \\
\pm \\
0.20\end{array}$ & $\begin{array}{l}0.57 \\
\pm \\
0.00\end{array}$ & $\begin{array}{l}0.56 \\
\pm \\
0.20\end{array}$ & $\begin{array}{l}0.58 \\
\pm \\
0.20\end{array}$ & $\begin{array}{l}0.75 \pm \\
0.00 *\end{array}$ & $\begin{array}{l}0.71 \pm \\
0.33^{*}\end{array}$ & +29.31 & -22.41 \\
\hline B & Bactofort $^{\circledR}$ & $\begin{array}{l}0.54 \\
\pm \\
0.10\end{array}$ & $\begin{array}{l}0.54 \\
\pm \\
0.25\end{array}$ & $\begin{array}{l}0.58 \\
\pm \\
0.20\end{array}$ & $\begin{array}{l}0.56 \\
\pm \\
0.00\end{array}$ & $\begin{array}{l}0.58 \\
\pm \\
0.10\end{array}$ & $\begin{array}{l}0.84 \pm \\
0.00 * \star\end{array}$ & $\begin{array}{l}0.80 \pm \\
0.00 * \star\end{array}$ & +44.83 & -37.93 \\
\hline C & $\begin{array}{l}\text { Positive } \\
\text { control }\end{array}$ & $\begin{array}{l}0.53 \\
\pm \\
0.10\end{array}$ & $\begin{array}{l}0.63 \\
\pm \\
0.30\end{array}$ & $\begin{array}{l}0.63 \\
\pm \\
0.33\end{array}$ & $\begin{array}{l}0.67 \\
\pm \\
0.10\end{array}$ & $\begin{array}{l}0.62 \\
\pm \\
0.20\end{array}$ & $\begin{array}{l}0.98 \pm \\
0.10 * \star \star\end{array}$ & $\begin{array}{l}0.94 \pm \\
0.00 * \star \star\end{array}$ & +58.06 & -51.61 \\
\hline D & $\begin{array}{l}\text { Negative } \\
\text { control }\end{array}$ & $\begin{array}{l}0.54 \\
\pm \\
0.00\end{array}$ & $\begin{array}{l}0.65 \\
\pm \\
0.25\end{array}$ & $\begin{array}{l}0.63 \\
\pm \\
0.20\end{array}$ & $\begin{array}{l}0.65 \\
\pm \\
0.30\end{array}$ & $\begin{array}{l}0.61 \\
\pm \\
0.10\end{array}$ & $\begin{array}{l}0.58 \pm \\
0.00\end{array}$ & $\begin{array}{l}0.57 \pm \\
0.20\end{array}$ & -4.92 & -6.56 \\
\hline
\end{tabular}

Key: $n=$ number of birds sampled, $S E=$ standard error of the mean, means with asterics $\left(^{*}\right)(P<0.05),\left({ }^{\star *}\right)(P<0.01)$, $(\star * *),(P<0.001)$ in the same column differed significantly

\section{Discussion}

The decrease in PCV, Hb, and RBC count could be as a result of non-regenerative anaemia which the vvIBDV caused by destruction of haemopoietic organ and viraemia in inoculated chicks (Campbell 1994; Mitchell and Johms 2008). But the decrease in these haematological parameters in chicks administered Antox ${ }^{\circledR}$ and Bactofort ${ }^{\circledR}$ was less severe, compared to that of positive control. Antox ${ }^{\circledR}$ and Bactofort ${ }^{\circledR}$ could have either enhanced production of erythropoietin, prevented the destruction of precursor cells in the bone marrow or the haemorrhages usually seen in IBD (Lukert and Saif, 2003; Kabir et al. 2004).

The decrease in TWBC, heterophil and lymphocyte counts which were lower in chicks administered Antox ${ }^{\circledR}$ and Bactofort ${ }^{\circledR}$ compared to positive control, is an indication that the probiotics possibly elicited the production of significant amount of immunoglobulins that neutralised the vvIBDV and thereby reduced the destruction of leucocytes (Midilli et al. 2008). The leucopaenia observed in positive control due to decrease in heterophils and lymphocytes counts at $7 \mathrm{dpi}$ was the consequence of corresponding heteropaenia and lymphopaenia. This result is in agreement with the findings of Cheville (1967), who also reported severe panleukopaenia during the severe inflammatory stage of IBD. The lymphopaenia observed in positive control at $7 \mathrm{dpi}$ was probably due to multiplication of vvIBDV in lymphocytes and subsequent necrosis of bursal lymphocytes as observed by Ley et al. (2007).

Heterophil/lymphocyte ratios provide important information of immune system tension following prolonged stress as well as infection status for some immunosuppressive diseases (Moreno et al. 2002; El Lethey et al. 2003; Clinchy et al. 2004). Heterophil/lymphocyte ratio was significantly higher in positive control when compared to that of the groups administered Antox ${ }^{\circledR}$ and Bactofort ${ }^{\circledR}$. Scope et al. (2002) observed a considerable increase in H/L ratio following stress associated with transporting, handling and viral diseases of birds. Acute stress is known to increase $\mathrm{H} / \mathrm{L}$ ratio (El Lethey et al. 2003). Therefore, the higher $\mathrm{H} / \mathrm{L}$ ratio observed in this study could be as a result of the inoculation of the chicks with the vvIBDV. 
Based on all the haematological parameters studied, Antox ${ }^{\circledR}$ mitigated the deleterious effects of vvIBDV better compared to Bactofort ${ }^{\circledR}$ in spite of the fact that it contained only one micro-organism (Saccharomyces cerevisiae). However, Antox ${ }^{\circledR}$ also contained vitamin B-complex, biotin, cobalt chloride, zinc, copper and iron chloride which are essential elements for the formation of red blood cells (Pimental 1992; Hochleithner 1994). Antox ${ }^{\circledR}$ also cannot just be considered as a probiotic only but rather serve as a source of nutritional supplement to take care of any deficiency in the commercial feed and the increase in demand four nutrients during IBDV infection. The efficacy the Bactofort ${ }^{\circledR}$ administered through feed may have also been adversely affected by the possible presence of antimicrobial agents in the commercial feed used in the present study.

From this study it was concluded that: Antox ${ }^{\circledR}$ and Bactofort ${ }^{\circledR}$ mitigated the deleterious effects of the vvIBDV inoculated on PCV, Hb, RBC, TWBC, lymphocyte and heterophil counts. Antox ${ }^{\circledR}$ and Bactofort ${ }^{\circledR}$ can therefore be used by farmers and veterinarians for the control of IBD.

\section{Declarations}

Funding: No funding was received by any of the authors for this research.

Conflict of interest: The authors declare no potential conflict of interest.

Availability of data and material: Not applicable

Code availability: Not applicable

Ethical approval: The ethics governing the use and conduct of experiments on animals were strictly observed, and the experimental protocol was approved by the Ahmadu Bello University Committee on Animal Use and Care (ABUCAUC) with the approval number ABUCAUC/2017/013.

\section{Author Contributions}

Conceptualization, supervision, review and editing - PAA and TA; methodology, investigation, data analyses, original draft preparation - ADA, FTA and OO. All authors have read and agreed to the published version of the manuscript.

Consent to participate: Not applicable

Consent for publication: Not applicable

\section{Acknowledgements}

Sampling of chicks and haematology would not have been possible without the kind assistance of Mr. Edima Abaja, Mr. David Leo of the Department of Veterinary Medicine and staff of the Clinical Pathology Laboratory of the Ahmadu Bello University Zaria, respectively.

\section{References}

1. Abdu PA (2014) Viral diseases. In Manual of Important Poultry Diseases in Nigeria 3rd edn (pp. 2-45). Jos, Nigeria: 6 and 4 Ventures.

2. Adamu J, Owoade AA, Abdu PA, Kazeem HM, Fatihu MY (2015) Characterization of field and vaccine infectious bursal disease viruses from Nigeria revealing possible virulence and regional marker in the VP2 minor hydrophilic peaks. Avian Pathol Doi:10.1080/030797.20133.822055. 
3. Amit-Romach E, Uni Z, Reifen R (2010) Multi-step mechanism of probiotics bacterium, the effect on innate immune system. Mol Food Nutr Res 54:277-284.

4. Becht H, Muller H (1991) Infectious bursal disease B-cell dependent immunodeficiency syndrome in chickens. Behring Inst Mitt 89:217-225.

5. Campbell TW (1994) Haematology. In B. W. Ritchie, G.J. Harrison \& L. R., Harrison (Eds). Avian Medicine: Principles and Application (176-198). Lake Worth: Wingers Publishing.

6. Campbell TW, Ellis CK (2007) Haematology of birds. In T.W Campbell \& C.K. Ellis (Eds.). Avian and Exotic Animal Haematology and Cytology 3rd edn (pp. 3-50). Ames: Blackwell Publishing Professional.

7. Cheville NF (1967) Studies on the pathogenesis of Gumboro disease in the bursa of Fabricius, spleen and thymus of the chicken. Am J Pathol 51:527-551.

8. Chineme CN, Cho Y (1984) Clinico-pathological and morphological chances in chickens experimentally infected with infectious bursal (Gumboro) disease virus. Trop Vet 2:218-224.

9. Christensen HR, Frokiaer H, Pestka JJ (2002) Lactobacilli differentially modulate expression of cytokines and maturation surface in murine dendritic cells. J Immunol 168:171-178.

10. Clinchy M, Zanette L, Boonstra R, Wingfield JC, Smith JNM (2004) Balancing food and predator pressure induces chronic stress in songbirds. In: Proceedings of the Royal Society of London. Series B: Biological Sciences (pp. 24732479).

11. Ehrmann MA, Kurzak P, Bauer J, Vogel RF (2002) Characterisation of Lactobacilli towards their use as probiotic adjuncts in poultry. J Appl Microbiol 92:966-975.

12. El Lethey H, Huber-Eicher B, Jungi TW (2003) Exploration of stress-induced immune-suppression in chickens reveals both stress-resistant and stress-susceptible antigen responses. Vet Immunol Immunopathol 95:91-101.

13. Fuller R (1989) Probiotics in man and animals. J Appl Bacteriol 66:365-378.

14. Glade MJ, Sist MO (1998) Dietary yeast culture supplementation enhances urea recycling in equine large intestine. Nutr Reprod Int 37:11-17.

15. Guillot JF (1998) Les probiotiques en alimentation animale. Cahiers Agricultures 7:49-54.

16. Hochleithner M (1994) Avian medicine: principles and application. In B.W. Ritchie, G.J. Harrison and L.R. Harrison (Eds.). Biochemistries (pp. 223-245). Wingers Publishing Inc., Lake Worth, FL.

17. Jackwood DJ, Saif YM, Hughes JH (1982) Characteristics and serologic studies of two serotypes of infectious bursal disease virus in turkeys. Avian Dis 26:82-871.

18. Kabir SML, Rahman MB, Rahman MM, Ahmed SU (2004) The dynamics of probiotics on growth performance and immune response in broilers. Int J Poult Sci 3:361-364.

19. Kibenge FS, Dhillon AS, Rusell RG (1988) Growth of serotypes I and II and variant strains of infectious bursal disease virus in vero cells. Avian Dis 32:298-303.

20. Lasher HN, Shane, SM (1994) Infectious bursal disease. World's Poult Sci J 50:133-166.

21. Ley K, Laudanna C, Cybulsky MI, Nourshargh S (2007) Getting to the site of information: the leukocyte adhesion cascade updated. Nature Rev Immunol 7:678-689.

22. Line EJ, Bailey SJ, Cox NA, Stern NJ, Tompkins T (1998) Effect of yeast - supplemented feed on Salmonella and Campylobacter populations in broilers. Poult Sci 77:405-410.

23. Lukert PD, Saif YM (1997) Infectious bursal disease. In B.W. Calnek, H.J. Barnes, C.W., Beard, L.R. McDougald \& Y.M. Saif (Eds.). Diseases of Poultry, 10th edn (pp. 721-738). Ames: lowa State University Press.

24. Lukert PD, Saif YM (2003) Infectious bursal disease. In Y.M. Saif, H.J. Barnes, J.R. Glisson, A.M. Fadly, L.R. McDougald, \& D.E. Swayne (Eds.). Diseases of Poultry11th edn (pp.161-179). Ames: lowa State Press.

Page $10 / 11$ 
25. Mcllroy SG, Goodall EA, McCracken RM (1989) Economic effects of sub-clinical infectious bursal disease on broiler production. Avian Pathol 18:465-480.

26. Midilli M, Alp M, Kocabagli N, Muglali OH, Turan N, Yilmaz H, Cakir S (2008) Effects of dietary probiotic and probiotic supplementation on growth performance and serum IgG concentration of broilers. South Afr J Animal Sci 38:21-27.

27. Mitchell EB, Johns J (2008) Avian haematology and related disorders. Vet Clinics Ex Animals Pract 11:501-522.

28. Moreno J, Merino S, Martinez J, Sanz JJ, Arriero E (2002) Heterophil/lymphocyte ratios and heat-shock protein levels are related to growth in nestling birds. Ecoscience 9:434-439.

29. Oladele OA, Adene DF, Obi TU, Nottidge HO, Aiyedun Al (2005) Sequential haematological study of experimental infectious bursal disease virus infection in chickens, turkeys and ducks. Revue D'elevage et de Medecine Veterinaire Des Pays Tropicaux 58:211-215.

30. Pagnini C, Saeed R, Bamias G, Arseneau KO, Pizarro TT, Cominelli F, Amit-Romach E, Uni Z, Reifen R (2010) Probiotics promote gut health through stimulation of epithelial innate immunity. Proceedings of the National Academy of Sciences (pp. 454-459),

31. Pimental JM, Greger JL, Cook MF, Stahl JL (1992) Iron metabolism in chicks fed various levels of zinc and copper. J Nutr Biochem 13:140-145.

32. Ross JG, Christie G, Halliday WG, Jones RM (1976) Determination of haematology and blood biochemistry values in healthy six weeks old broiler hybrids. Avian Pathol 5:273-281.

33. Scope A, Filip T, Gabler C, Resch F (2002) The influence of stress from transport and handling on haematologic and clinical chemistry blood parameters of racing pigeons (Columba livia domestica). Avian Dis 46:224-229.

34. Sharma JM, Ki LJ, Rautenschlein S, Yeh HY (2000) Infectious bursal disease virus of chicken: Pathogenesis and immunosuppression. Dev Comp Immunol 24:223-235. 